\title{
Optimal consumption/investment problem with light stocks: A mixed continuous-discrete time approach
}

\author{
Rosella Castellano ${ }^{\mathrm{a}}$, Roy Cerqueti ${ }^{\mathrm{b}, *}$ \\ ${ }^{a}$ Department of Economic and Financial Institutions, University of Macerata, Italy \\ ${ }^{\mathrm{b}}$ Department of Economic and Financial Institutions, University of Macerata, Via Crescimbeni 20, 62100 Macerata, Italy
}

\section{A R T I C L E I N F O}

\section{Keywords:}

Optimal consumption/investment model

Utility maximization

Thin stocks

Stochastic control theory

Dynamic programming

Jump-diffusion dynamics

Monte Carlo simulations

\begin{abstract}
A B S T R A C T
This paper addresses the optimal consumption/investment problem in a mixed discrete/ continuous time model in presence of rarely traded stocks. Stochastic control theory with state variable driven by a jump-diffusion, via dynamic programming, is used. The theoretical study is validated through numerical experiments, and the proposed model is compared with the classical Merton's portfolio. Some financial insights are provided.
\end{abstract}

(c) 2011 Elsevier Inc. All rights reserved.

\section{Introduction}

In finance one of the most debated issues is the optimal asset allocation, which is particularly relevant both by a theoretical and a practical perspective.

The pioneer of portfolio theory is [10], which proposes a single period model with normally distributed risky returns and absence of transaction costs. The architecture of the Markowitz's model has been reviewed in a more realistic fashion by the Markowitz's followers.

Samuelson [19] extends the original uniperiodal framework to a multiperiod setting, while $[12,13]$ deal with a continuous time portfolio model.

Continuous time portfolio models have been improved by the introduction of random jumps in the dynamics of the risky assets (see [1,8]) where, in some cases, the presence of jumps depends on transaction costs (see [9]).

In this paper, the optimal consumption/investment problem is addressed in a mixed continuous-discrete-time model, in order to consider rarely traded assets.

The frequency of trade is a measure of the stock liquidity, so that an infrequently traded stock is associated to low liquidity. Hereafter, we refer to thin or light stocks as synonymous of rarely traded assets.

The problem discussed here is relevant both by a theoretical and a financial point of view. By a financial perspective, the presence of thin securities is a widespread phenomenon which becomes even more relevant when risky assets of emerging economies are considered. In this regard, it is worth noting that significant relations between low trading volumes and low market quality (i.e. wide bid/ask spreads, high volatility, low informative efficiency, high adverse selection costs), documented by several empirical studies (see [7]), become even more important in times of financial crisis. By a mathematical perspective, continuous-time hypothesis may realistically describe the dynamics of a high-liquidity risky asset, but it becomes unreasonable when thinly traded assets are taken into account. Therefore the introduction of discrete-time random

\footnotetext{
* Corresponding author.

E-mail address: roy.cerqueti@unimc.it (R. Cerqueti).
} 
dynamics for the returns of thin stocks is required. In this paper random trading times are considered, and this leads to a very complex model.

Some other papers discuss the problem highlighted above. Matsumoto [11] introduces trades at random times following a Poisson law, but consumption is not taken into account. Rogers [17] introduces discrete random times, but consumption is assumed to be constant between trading dates. In the stochastic optimal control problem of [18], the authors introduce consumption with a rate changing between trading dates, but the optimal consumption policy is not derived in a closed form. Cretarola et al. [4] limits its analysis to the case of choice between illiquid assets and consumption.

The contribution of this paper relies on the presence of a time dependent stochastic consumption, in a financial setting with riskless bonds, frequently traded risky assets and thin stocks. In doing so, the papers quoted above are extended in many directions. According with [4], we adopt the model developed by Pham and Tankov [15] for describing the dynamics of infrequently traded assets. In particular, jumps are modeled by using a Levy process, which is particularly appropriate for this purpose (see $[2,3,5,6,20]$ ).

Stochastic control theory, in a dynamic programming framework with jump diffusions, is the followed approach. For a survey on stochastic control theory with state variables driven by jump diffusions, see [14].

The value function of the control problem is given by the maximized discounted expected utility of the investor. The problem is first theoretically solved in a very general setting; then, focusing on a particular power-type utility function, the optimal strategies in explicit closed form are derived. The solving strategy is in line with the approach adopted by Shin et al. [21], which solves a general consumption/investment problem with downside constraints and uses a CRRA utility function to deal with the numerical validation of the theoretical model.

The optimal strategies are then compared to the ones of the classical [13]'s model. Such a comparison is particularly interesting, since Merton's seminal work deals with an optimal consumption/investment problem where there are not opportunities to invest in low-liquidity stocks. The numerical validation of the theoretical model provides insights on the optimal strategies and paths in relation to the frequency of the trading dates in the thin stock.

The paper is organized as follows. Next section presents the development of the model in a general framework. Section 3 provides the analysis of the optimal strategies with a power-type utility function. Section 4 is devoted to some numerical experiments and presents the comparison between the proposed theoretical model and Merton's one. Last section concludes.

\section{Model development in a general setting}

In this section the economic framework of the model is presented. All the random quantities defined throughout the paper are assumed to be contained in a probability space with filtration $\left(\Omega, \mathcal{F},\left\{\mathcal{F}_{t}\right\}_{t \geqslant 0}, P\right)$, where the filtration $\mathcal{F}_{t}$ is assumed to reflect the whole set of information provided by the market up to time $t$. The investor shares her/his wealth among three assets, i.e. a risk free bond, a liquid risky asset and a light stock:

- the price of the riskless bond $B_{t}$ evolves according to the following ordinary differential equation:

$$
d B_{t}=r(t) B_{t} d t, \quad t \geqslant 0,
$$

where $r(t)$ is the deterministic continuously compounded risk free interest rate at time $t$;

- the price of the risky liquid asset $S_{t}$ evolves as follows:

$$
d S_{t}=\mu_{1} S_{t} d t+\sigma_{1} S_{t} d W_{t}^{1}, \quad t \geqslant 0
$$

where the expected rate of return $\mu_{1}$ is greater than $r(t)$, for each $t \geqslant 0$, and $\sigma_{1}$ is the instantaneous standard deviation of the rate of return; $W^{1}$ is a standard 1-dimensional Brownian Motion;

- the price of the thin stock $H_{t}$ is assumed to follow a geometric Brownian Motion:

$$
d H_{t}=\mu_{2} H_{t} d t+\sigma_{2} H_{t} d W_{t}^{2}, \quad t \geqslant 0,
$$

where the expected rate of return $\mu_{2}$ is greater than $r(t)$, for each $t \geqslant 0$, and $\sigma_{2}$ is the instantaneous standard deviation of the rate of return of the light stock; $W^{2}$ is a standard 1-dimensional Brownian Motion.

By definition of thin stock, it is realistically assumed that $\mu_{2}>\mu_{1}$ and $\sigma_{2}>\sigma_{1}$.

The financial characteristics of the thin stock imply that the dynamics of its returns should be modeled by a jump-type process. The model proposed by Pham and Tankov [15] is adopted, and it is assumed that investors can trade the thin stock only at random times $\left\{\tau_{k}\right\}_{k \geqslant 0}$, with $\tau_{0}=0<\tau_{1}<\cdots<\tau_{k}<\cdots$. We denote by $Z_{k}$ the stochastic return of the light stock in the random time interval $\tau_{k}-\tau_{k-1}$, for each $k \in \mathbb{N}$ : 


$$
Z_{k}=\frac{H_{\tau_{k}}-H_{\tau_{k-1}}}{H_{\tau_{k-1}}}
$$

In this market, the agent holds a capital to be shared among the above described three assets. Moreover, a part of the capital goes to consumption. The choices of the agent change with time. More specifically, we denote by $\eta, \theta, \zeta, c$ four stochastic processes representing the shares of capital invested on the riskless bond, the risky asset and the thin stock and the amount of consumption, respectively. Since the thin stock faces discrete random returns, also $\zeta$ should have realizations in the discrete times $\tau$ 's. More precisely, $\zeta_{t}=0$, for $t \notin\left\{\tau_{n}\right\}_{n \in \mathbb{N}}$. We will denote $\zeta_{\tau_{i}}=: \zeta_{i}$, for $i \in \mathbb{N}$

Assuming that $\eta_{t}+\theta_{t}+\zeta_{t}=1$, for each $t \geqslant 0$, the portfolio is determined by a self-financing trading strategy with consumption. Hence, the portfolio wealth $X_{t}$ of the agent at time $t \geqslant 0$ can be written as follows:

$$
X_{t}=x+\int_{0}^{t}\left\{X_{s}\left[\left(1-\theta_{s}-\zeta_{s}\right) r(s)+\mu_{1} \theta_{s}\right]-c_{s}\right\} d s+\int_{0}^{t} \sigma_{1} X_{s} \theta_{s} d W_{s}^{1}+\sum_{i=1}^{+\infty} X_{\tau_{i}} \zeta_{i} Z_{i} \mathbf{1}_{\left\{\tau_{i} \leqslant t\right\}},
$$

where $X_{0}=x>0$ is the initial wealth. The point process $\left\{\left(\tau_{i}, Z_{i}\right)\right\}_{i}$ is assumed to be given by the jumps of a Lévy process $\Gamma_{t}$. We do not lose of generality by assuming that $\Gamma_{t}$ is cadlag. In this sense, a jump at time $t$ is described by $\Delta \Gamma_{t}=\Gamma_{t}-\Gamma_{t-}$.

Consider a Borel set $\mathcal{B}$ in $\mathbb{R}$. The number of jumps occurring in the period $[0, t]$ with size in $\mathcal{B}$ can be written as follows:

$$
N(t, \mathcal{B})=\sum_{i=1}^{+\infty} \sharp_{\mathcal{B}}\left(\Delta \Gamma_{\tau_{i}}\right) \mathbf{1}_{\left\{\tau_{i}<t\right\}},
$$

where, for each $i \in \mathbb{N}$, we define

$$
\sharp_{\mathcal{B}}\left(\Delta \Gamma_{\tau_{i}}\right):= \begin{cases}1, & \text { if } \Delta \Gamma_{\tau_{i}} \in \mathcal{B} ; \\ 0, & \text { otherwise. }\end{cases}
$$

By substituting the discrete process $Z$ with the continuous version $\Gamma$, we can rewrite (5) as follows:

$$
X_{t}=x+\int_{0}^{t}\left\{X_{s}\left[\left(1-\theta_{s}-\zeta_{s}\right) r(s)+\mu_{1} \theta_{s}\right]-c_{s}\right\} d s+\int_{0}^{t} \sigma_{1} X_{s} \theta_{s} d W_{s}^{1}+\int_{0}^{t} \int_{-1}^{+\infty} X_{s} \zeta_{s} z N(d s, d z)
$$

where $N(d s, d z)$ is the differential of $N(t, \mathcal{B})$.

The following assumption holds true hereafter.

Assumption 1. $\left\{\tau_{i}\right\}_{i \in \mathbb{N}}$ is a sequence of jumps of a Poisson process with intensity $\lambda$.

Since the point process $\left\{\left(\tau_{i}, Z_{i}\right)\right\}_{i}$ is a Levy process, then $Z_{k}$ is independent from $\left\{\left(\tau_{i}, Z_{i}\right)\right\}_{i<k}$. We denote its distribution as $p(t, d z)$, where $t=\tau_{k}-\tau_{k-1}>0$.

The following result is particularly important for the development of the model. For the proof, we refer to [16, Theorem 1.35].

Theorem 2. Consider a Borel set $\mathcal{B}$ in $\mathbb{R}$ and define

$$
Y(t):=\sum_{i=1}^{+\infty} Z_{i} \mathbf{1}_{\left\{\tau_{i} \leqslant t\right\}}
$$

The Levy measure $v$ of $Y(t)$ is given by

$$
v(\mathcal{B})=\mathbb{E}[N(1, \mathcal{B})]=\lambda p(1, \mathcal{B}),
$$

where $\mathbb{E}$ is the usual expected value operator.

The following assumption will stand in force hereafter:

\section{Assumption 3}

$$
\int_{-1}^{+\infty}(1 \wedge|z|) v(d z)<+\infty
$$

The validity of Assumption 3 implies that jumps have finite variation.

Let us define the admissible control processes.

Definition 4. An admissible control policy is a triplet $(\theta, \zeta, c)$ of continuous Markovian time processes such that

$$
\begin{aligned}
& \theta:[0,+\infty) \times \Omega \rightarrow \mathbb{R} \text { such that } \theta_{t} \in \mathcal{F}_{t}, \\
& \zeta:[0,+\infty) \times \Omega \rightarrow \mathbb{R} \text { such that } \zeta_{t} \in \mathcal{F}_{t}, \\
& c:[0,+\infty) \times \Omega \rightarrow[0,+\infty) \text { such that } c_{t} \in \mathcal{F}_{t} .
\end{aligned}
$$


The admissible control policies live in the admissible region, which depends on the initial data $x$. We denote it as $\mathcal{A}(x)$.

The investor aims at searching for the best consumption/investment combination to pursue the maximization of her/his discounted expected utility. The objective functional $J$ is defined as follows:

$$
J(x, \theta, \zeta, c):=\mathbb{E}_{x}\left[\int_{0}^{+\infty} e^{-\rho t} U\left(c_{t}\right) d t\right], \quad x>0,
$$

where $e^{-\rho}$ is an unitary discount factor, $U$ is the utility function defined in $[0,+\infty)$ and $\mathbb{E}_{x}$ indicates the conditional expectation given $X_{0}=x$.

The value function of the optimal consumption/portfolio problem is written as the maximization of the expected discounted utility as follows:

$$
V(x):=\sup _{(\theta, \xi, c) \in \mathcal{A}(x)} \mathbb{E}_{x}\left[\int_{0}^{+\infty} e^{-\rho t} U\left(c_{t}\right) d t\right], \quad x>0 .
$$

We now state some conditions on the utility function $U$.

Assumption 5. $U \in C^{1}(0,+\infty)$, it is strictly increasing, strictly concave, $U(0)=0$ and the Inada conditions are satisfied, i.e.

$$
\lim _{c \rightarrow 0^{+}} U^{\prime}(c)=+\infty ; \quad \lim _{c \rightarrow+\infty} U^{\prime}(c)=0 .
$$

As also argued in [4], Assumption 5 does not provide a strong restriction on the choice of the utility function $U$, since the most common utility functions satisfy it.

To develop the model, it is necessary to investigate the properties of the value function.

A direct consequence of Assumption 5 is stated in the next result (see [14, Re mark 3.3]).

Proposition 6. $V$ is strictly concave in $(0,+\infty)$.

By using the concavity of $V$, we derive the following:

Proposition 7. $V$ is continuous in $(0,+\infty)$.

For the proof, see the Appendix.

Since $(\theta, \zeta, c)$ is a triple of Markov controls, then the generator of the diffusion Levy process $X_{t}$ is

$$
A_{\theta, \zeta, c} \phi(x)=\left\{x\left[(1-\theta-\zeta) r+\mu_{1}\right]-c\right\} \phi^{\prime}(x)+\frac{\sigma_{1}^{2} x^{2} \theta^{2}}{2} \phi^{\prime \prime}(x)+\int_{-1}^{+\infty}\left\{\phi(x+z x \zeta)-\phi(x)-z x \zeta \phi^{\prime}(x)\right\} v(d z),
$$

where the Levy measure $v$ describes the jumps of the process $X$.

The Hamilton Jacobi Bellman equation (HJB) can be stated by applying Ito's Lemma.

Theorem 8 (HJB). Assume that $V \in C^{2}(0,+\infty)$. Then

$$
\rho V(x)=\sup _{(\theta, \zeta, c) \in \mathbb{R}^{2} \times[0,+\infty)}\left[U(c)+A_{\theta, \zeta, c} V(x)\right] .
$$

Theorem 8 assures that the value function is a formal solution of the HJB (14): indeed, we have to impose the right regularity of the value function in order to derive Eq. (14). In most of the cases, the value function is not smooth enough. Further discussions on this topic will be addresses in the next section.

The optimal strategy and trajectory can be theoretically identified by using a Verification Theorem. We do not need to provide an original formulation for this result, since the proposed model is a particular case of the very general setting proved in [14, Theorem 8.1]. Therefore, the adaptation of the Verification Theorem to this framework is enunciated, while details are left to the quoted reference.

Theorem 9 (Verification Theorem). Assume that $u \in C^{2}((0,+\infty))$ is a classical solution of the HJB (14).

Then we have

(a) $u(x) \geqslant V(x), \quad \forall x \in(0,+\infty)$.

(b) Let us consider $\left(\theta^{*}, \zeta^{*}, c^{*}\right)$ an admissible triple and $X^{*}$ a trajectory starting at $x$ such that

$$
\left(\theta^{*}, \zeta^{*}, c^{*}\right) \in \operatorname{argmax}_{\theta, \zeta, c}\left\{U(c)+A_{\theta, \zeta, c} u(x)\right\} .
$$

Then $\left(\theta^{*}, \zeta^{*}, c^{*}\right)$ is optimal, with optimal path $X^{*}(t)$ at $x$, if and only if $u(x)=V(x), \forall x \in(0,+\infty)$.

The next step of this work is to provide an explicit form for the optimal strategies and trajectories. By separating the control parameters, Eq. (14) can be rewritten as follows: 


$$
\begin{aligned}
& \rho V(x)-x r V^{\prime}(x)+\int_{-1}^{+\infty} V(x) v(d z)-\sup _{c \in[0,+\infty)}\left[U(c)-c V^{\prime}(x)\right]-\sup _{\theta \in \mathbb{R}}\left[x \theta\left(\mu_{1}-r\right) V^{\prime}(x)+\frac{\sigma_{1}^{2} x^{2} \theta^{2}}{2} V^{\prime \prime}(x)\right] \\
& -\sup _{\zeta \in \mathbb{R}}\left[-x r \zeta V^{\prime}(x)+\int_{-1}^{+\infty}\left\{V(x+z x \zeta)-z x \zeta V^{\prime}(x)\right\} v(d z)\right]=0 .
\end{aligned}
$$

The value optimizing the operator appearing in the right-hand side of (14) is formalized in the next result.

Proposition 10. Fix $x \in(0,+\infty)$ and define:

$$
c^{*}(x):=\arg \max _{c \in \mathbb{R}}\left[U(c)-c V^{\prime}(x)\right]=I\left(V^{\prime}(x)\right),
$$

where I is the inverse $e^{1}$ of the first derivative of $U$;

$$
\begin{aligned}
& \theta^{*}(x):=\arg \max _{\theta \in \mathbb{R}}\left[x \theta\left(\mu_{1}-r\right) V^{\prime}(x)+\frac{\sigma_{1}^{2} x^{2} \theta^{2} V^{\prime \prime}(x)}{2}\right]=\frac{-x\left(\mu_{1}-r\right) V^{\prime}(x)}{\sigma_{1}^{2} x^{2} V^{\prime \prime}(x)} ; \\
& \zeta^{*}(x):=\arg \max _{\zeta \in \mathbb{R}}\left[-x r \zeta V^{\prime}(x)+\int_{-1}^{+\infty}\left\{V(x+z x \zeta)-z x \zeta V^{\prime}(x)\right\} v(d z)\right] .
\end{aligned}
$$

The triple $\left(\theta^{*}(x), \zeta^{*}(x), c^{*}(x)\right)$ fulfils the maximization in Eq. (14), i.e.

$$
\sup _{(\theta, \zeta, c) \in \mathbb{R}^{2} \times[0,+\infty)}\left[U(c)+A_{\theta, \zeta, c} V(x)\right]=U\left(c^{*}(x)\right)+A_{\theta^{*}(x), \xi^{*}(x), c^{*}(x)} V(x) .
$$

Proof. The proof is straightforward, and moves from the concavity of the function $V$ (see Proposition 6).

The connection between (16)-(18) and the couple (optimal control, optimal trajectory) can be observed through the introduction of the closed loop equation:

$$
X_{t}=x+\int_{0}^{t}\left\{X_{s}\left[\left(1-\theta\left(X_{s}\right)-\zeta\left(X_{s}\right)\right) r(s)+\mu_{1} \theta\left(X_{s}\right)\right]-c\left(X_{s}\right)\right\} d s+\int_{0}^{t} \sigma_{1} X_{s} \theta\left(X_{s}\right) d W_{s}^{1}+\sum_{i=1}^{+\infty} X_{\tau_{i}} \zeta\left(X_{\tau_{i}}\right) Z_{i} \mathbf{1}_{\left\{\tau_{i} \leqslant t\right\}} .
$$

In the next result, the significance of the closed loop equation is shown, together with the optimal strategies and the optimal trajectory.

Proposition 11. Consider $x \in(0,+\infty)$ and the admissible controls $c^{*}(x), \theta^{*}(x), \zeta^{*}(x)$ formalized in Proposition 10.

Denote as $\bar{X}$ the solution of the closed loop equation

$$
\bar{X}_{t}=x+\int_{0}^{t}\left\{\bar{X}_{s}\left[\left(1-\theta^{*}\left(\bar{X}_{s}\right)-\zeta^{*}\left(\bar{X}_{s}\right)\right) r(s)+\mu_{1} \theta^{*}\left(\bar{X}_{s}\right)\right]-c^{*}\left(\bar{X}_{s}\right)\right\} d s+\int_{0}^{t} \sigma_{1} \bar{X}_{s} \theta^{*}\left(\bar{X}_{s}\right) d W_{s}^{1}+\sum_{i=1}^{+\infty} \bar{X}_{\tau_{i}} \zeta^{*}\left(\bar{X}_{\tau_{i}}\right) Z_{i} \mathbf{1}_{\left\{\tau_{i} \leqslant t\right\}} .
$$

Then, setting $\bar{c}_{t}=c^{*}\left(\bar{X}_{t}\right), \bar{\theta}_{t}=\theta^{*}\left(\bar{X}_{t}\right)$ and $\bar{\zeta}_{t}=\zeta^{*}\left(\bar{X}_{t}\right)$, we have $J(x, \bar{c}, \bar{\theta}, \bar{\zeta})=V(x)$, and the triple $(\bar{c}, \bar{\theta}, \bar{\zeta})$ is optimal for the control problem. The optimal path is $\bar{X}_{t}$.

As stressed above, generally, the value function is not twice differentiable, and the HJB (14) does not admit a classical solution. Therefore, the problems related with the existence and uniqueness of the solution of the integro-differential equation (14) and the regularity properties of the value function need to be addressed. This is the subject of the next section.

\section{Explicit derivation of the optimal strategies with power-type utility function}

Rather than discussing the regularity of the value function only by a theoretical perspective, we solve this issue by using a particular power-type utility function $U$, in order to compare the proposed model to the classical [13]'s one.

Assume a power-type utility function $U$ satisfying Assumption 5, i.e. there exists $\gamma \in(0,1)$ such that

$$
U(c)=\frac{c \gamma}{\gamma}
$$

Then we try a value function of power-type, with the same exponent:

$$
V(x)=K x^{\gamma},
$$

with an opportune value of $K \in[0,+\infty)$.

Since $V \in C^{2}(0,+\infty)$, Theorem 8 guarantees that $V$ satisfies Eq. (14), which can be rewritten as:

\footnotetext{
${ }^{1}$ The existence of $I$ is assured by the hypothesis of strict increasingness and concavity of $U$.
} 


$$
\rho K x^{\gamma}=\sup _{(\theta, \zeta, c) \in \mathbb{R}^{2} \times[0,+\infty)}\left[\frac{c \gamma}{\gamma}+K \gamma x^{\gamma-1}\left\{\left[(1-\theta-\zeta) r+\mu_{1} \theta\right] x-c\right\}+\frac{K \gamma(\gamma-1) \sigma_{1}^{2} \theta^{2} x^{\gamma}}{2}+K x^{\gamma} \int_{-1}^{+\infty}\left\{(1+z \zeta)^{\gamma}-1-\gamma z \zeta\right\} v(d z)\right] .
$$

Denote as $H(\theta, \zeta, c)$ the argument of the sup operator in squared brackets, in the right hand side of (24).

To obtain the optimal strategies, we move in accord to (16)-(18). Since $H$ is a concave operator, the stationary points of $H$ are the optimal strategies, and only the first order conditions are needed.

$$
\left\{\begin{array}{l}
\frac{\partial H(\theta, \zeta, c)}{\partial c}=c^{\gamma-1}-K \gamma x^{\gamma-1}=0, \\
\frac{\partial H(\theta, \zeta, c)}{\partial \theta}=K \gamma\left[\mu_{1}-r+(\gamma-1) \sigma_{1}^{2} \theta\right] x^{\gamma}=0, \\
\frac{\partial H(\theta, \zeta, c)}{\partial \zeta}=K \gamma x^{\gamma}\left[-r+\int_{-1}^{+\infty} z\left\{(1+z \zeta)^{\gamma-1}-1\right\} v(d z)\right]=0 .
\end{array}\right.
$$

Now, define the function

$$
\Lambda(\zeta)=-r+\int_{-1}^{+\infty} z\left\{(1+z \zeta)^{\gamma-1}-1\right\} v(d z) .
$$

Note that $\Lambda$ is continuous. Moreover,

$$
\Lambda(0)=-r<0 ; \quad \lim _{\zeta \rightarrow+\infty} \Lambda(\zeta)=+\infty .
$$

Therefore, there exists $\zeta^{*} \in(0,+\infty)$ such that $\Lambda\left(\zeta^{*}\right)=0$. Hence, fixed $x \in(0,+\infty)$, by (25), the optimal strategies are $\left(c^{*}(x), \theta^{*}(x), \zeta^{*}(x)\right)$, where

$$
\left\{\begin{array}{l}
c^{*}(x)=\left(K \gamma x^{\gamma-1}\right)^{\frac{1}{\gamma-1}} \\
\theta^{*}(x)=\theta^{*}=\frac{r-\mu_{1}}{(\gamma-1) \sigma_{1}^{2}} \\
\zeta^{*}(x)=\zeta^{*} \text { such that } \quad-r+\int_{-1}^{+\infty} z\left\{\left(1+z \zeta^{*}\right)^{\gamma-1}-1\right\} v(d z)=0 .
\end{array}\right.
$$

By substituting the optimal strategies of (27) into (24) we obtain the value of $K$ as:

$$
K=\left\{\frac{\rho-\gamma\left[\left(1-\theta^{*}-\zeta^{*}\right) r+\mu_{1} \theta^{*}\right]-\frac{\gamma(\gamma-1) \sigma_{1}^{2}\left(\theta^{*}\right)^{2}}{2}-\int_{-1}^{+\infty}\left\{\left(1+z \zeta^{*}\right)^{\gamma}-1-\gamma z \zeta^{*}\right\} v(d z)}{\gamma^{\frac{\gamma}{\gamma-1}}\left(\frac{1}{\gamma}-1\right)}\right\}^{\gamma-1} .
$$

Therefore, the power-type value function (23), with $K$ given by (28), satisfies (24).

The system of optimal control-optimal trajectory may be derived by Proposition 11 through the closed loop equation given in (20).

Proposition 12. Consider $x \in(0,+\infty)$ and the optimal strategies $c^{*}(x), \theta^{*}(x), \zeta^{*}(x)$ given in (27).

Denote as $\bar{X}$ the solution of the closed loop equation

$$
\bar{X}_{t}=x+\int_{0}^{t}\left\{\bar{X}_{s}\left[\left(1-\theta^{*}\left(\bar{X}_{s}\right)-\zeta^{*}\left(\bar{X}_{s}\right)\right) r(s)+\mu_{1} \theta^{*}\left(\bar{X}_{s}\right)\right]-c^{*}\left(\bar{X}_{s}\right)\right\} d s+\int_{0}^{t} \sigma_{1} \bar{X}_{s} \theta^{*}\left(\bar{X}_{s}\right) d W_{s}^{1}+\sum_{i=1}^{+\infty} \bar{X}_{\tau_{i}} \xi^{*}\left(\bar{X}_{\tau_{i}}\right) Z_{i} \mathbf{1}_{\left\{\tau_{i} \leqslant t\right\}} .
$$

Then, setting $\bar{c}_{t}=c^{*}\left(\bar{X}_{t}\right), \bar{\theta}_{t}=\theta^{*}\left(\bar{X}_{t}\right)$ and $\bar{\zeta}_{t}=\zeta^{*}\left(\bar{X}_{t}\right)$, we have $J(x, \bar{c}, \bar{\theta}, \bar{\zeta})=V(x)$, and the quadruple $(\bar{X}, \bar{c}, \bar{\theta}, \bar{\zeta})$ is optimal for the control problem.

By Proposition 12 we can write explicitly the optimal strategies:

$$
\left\{\begin{array}{l}
\bar{c}_{t}=\left(K \gamma \bar{X}_{t}^{\gamma-1}\right)^{\frac{1}{\gamma-1}} \\
\bar{\theta}=\frac{r-\mu_{1}}{(\gamma-1) \sigma_{1}^{2}} \\
\bar{\zeta} \quad \text { such that } \quad-r+\int_{-1}^{+\infty} z\left\{(1+z \bar{\zeta})^{\gamma-1}-1\right\} v(d z)=0
\end{array}\right.
$$

\section{Numerical analysis}

The purpose of this section is to provide, via numerical analysis, insights on the optimal strategies and paths which are explicitly stated for the power-type utility function described in Section 3.

The analysis of several cases is provided. Firstly, the frequency of the jumps in thin stock returns is taken into account, in order to state the relationship between optimal paths and trading dates characterizing the low-liquidity asset. Secondly, the relationship between the optimal consumption and the optimal portfolio wealth is investigated. Thirdly, the comparison between the proposed model and the one in [13] is performed. 
The general setting of the application can be synthesized as follows: the investment horizon is 1 year, i.e. $T=252$ business days; the constant relative coefficient of risk aversion is $1-\gamma=0.1$, i.e. $\gamma=0.9$; the investor allocates funds in a liquid and continuously traded risky asset with expected rate of return $\mu_{1}=0.08$ and volatility $\sigma_{1}=0.15$, in a light stock not continuously traded (illiquid) with expected rate of return $\mu_{2}=0.2$ and volatility $\sigma_{2}=0.3$ and in a riskless bond with constant rate of return $r=0.03$; the Poisson law parameter $\lambda$ is alternatively set to $5,15,30,60$ and 100 . It is worth to notice that the average frequency of the jumps in light stock returns is inversely proportional to the magnitude of $\lambda$.

The first building block of the numerical procedure is represented by the simulation of the random returns, at random times, of the thin stock, according to Eqs. (3) and (4).

The general iterative method for simulating random times and random returns of the thin stock consists of the following steps:

- set $t_{0}=0$ and $H_{0}=0.40$;

- for $j=1,2, \ldots$, generate a random variable $R_{j+1}$ from the exponential distribution with mean $1 / \lambda$;

- set $t_{j+1}=t_{j}+R_{j+1}$;

- generate $B_{j+1} \sim N(0,1)$;

- set $H_{j+1}=H_{j}\left(1+\mu_{2} R_{j+1}+\sigma_{2} \sqrt{R_{j+1}} B_{j+1}\right)$;

- set $z_{j+1}=\frac{H_{j+1}-H_{j}}{H_{j}}$;

- if $t_{j+1}>1$, stop. We denote the last index as $j^{*}$, i.e. $t_{j^{*}} \leqslant 1$ and $t_{j^{*}+1}>1$.

The procedure described above is replicated $n=10,000$ times. Obviously, the value of $j^{*}$ depends on the replication index, $i$. Denote as $j^{*}(i)$ the value of the last index $j^{*}$ for the $i$ th replication. The returns of the thin stocks can be allocated in an incomplete matrix $\mathcal{Z}=\left(z_{j}^{(i)}\right)_{j=1, \ldots j^{*}(i) ; i=1, \ldots, n}$, corresponding to an incomplete matrix of random times $\mathcal{T}=\left(t_{j}^{(i)}\right)_{j=1, \ldots . j^{*}(i) ; i=1, \ldots, n}$. The returns of the thin stock are computed as a particular mean of the values obtained with $n$ replications as follows: first, the elements of the matrix $\mathcal{T}$ are clustered in the $T=252$ time-intervals, representing the business days; second, the corresponding returns are accordingly clustered; third, the returns of the thin stocks are obtained as weighted average of the returns falling in each cluster. More formally, we obtain a return vector $\mathbf{Z}=\left(Z_{1}, \ldots, Z_{T}\right)$, where:

$$
Z_{j}=\frac{1}{n} \sum_{i=1}^{n} z_{k}^{(i)} \cdot \mathbf{1}_{t_{k}^{(i)} \in\left[\frac{j-1}{T}, T\right]}, \quad j=1, \ldots, T .
$$

The second building block of the application consists in writing the optimal control triple of the problem as stated in (27). At this aim, we set the value of the portfolio at time 0 as $x=1$. While $\theta^{*}$ and $c^{*}(x)$ are defined as in the first and the second formulas of (27), the value of $\zeta^{*}$ is derived from the solution of the third equation in (27). To find the optimal $\zeta^{*}$, a grid search algorithm is implemented as follows:

- set the range of $\zeta^{*}$ as $[-1,1]$;

- discretize the range $[-1,1]$ with step $\frac{1}{10,000}$;

- set the vector $\left(\zeta_{1}^{*}, \ldots, \zeta_{m}^{*}\right)$, with $m=2 \times 10,000$, where $\zeta_{h}^{*}=-1+h \cdot \frac{1}{10,000}$;

- set the range of the returns of the thin stock as $[-1,2]$;

- discretize the range $[-1,2]$ with step $\frac{1}{100}$ and obtain $[-1,2]=\mathcal{I}_{1} \cup \ldots \cup \mathcal{I}_{l}$, where $l=3 \times 100$ and $\mathcal{I}_{k}=\left[-1+\frac{k-1}{l},-1+\frac{k}{l}\right]$;

- take the returns $z_{j}^{(i)}$ computed in the first building block, with $i=1, \ldots, n$ and $j=1, \ldots, j^{*}(i)$;

- for each replication $i$, discretize the differential term $v(d z)$ as:

$$
v^{(i)}\left(\mathcal{I}_{k}\right)=\sum_{j=1}^{j^{*}(i)} \sharp_{\mathcal{I}_{k}}\left(z_{j}^{(i)}\right),
$$

where the operator $\sharp$. is defined as in (7);

- compute the weighted average with respect to the number of replications $i$ and the $z$ 's, and define:

$$
v\left(\mathcal{I}_{k}\right)=\frac{1}{n} \sum_{i=1}^{n} v^{(i)}\left(\mathcal{I}_{k}\right)
$$

and

$$
z_{k}=\frac{1}{n} \sum_{i, j} z_{j}^{(i)} \mathbf{1}_{z_{j}^{(i)} \in \mathcal{I}_{k}} ;
$$

- define the vector of discretized integral as $\left(\Gamma_{1}, \ldots, \Gamma_{n}\right)$, where:

$$
\Gamma_{h}=\sum_{k=1}^{l} z_{k}\left[\left(1+z_{k} \zeta_{h}\right)^{\gamma-1}-1\right] v\left(\mathcal{I}_{k}\right) ;
$$


- find the optimal $\zeta^{*}=\zeta_{h^{*}}$, where $h^{*}$ is selected to have:

$$
\left|\Gamma_{h^{*}}-r\right|=\min _{h=1, \ldots, m}\left|\Gamma_{h}-r\right| \text {. }
$$

The third building block of the application is related with the substitution of the optimal strategies $c^{*}(x), \theta^{*}$ and $\zeta^{*}$ into the dynamics of the portfolio wealth, accordingly to the closed loop equation (29). Then, by using Proposition 12, the optimal control triple and the optimal path are derived.

We perform $n=10,000$ replications of the following algorithm:

- set $x=1$;

- set $\mu=\left(1-\theta^{*}-\zeta^{*}\right) r+\mu_{1} \theta^{*}$;

- set $\sigma=\sigma_{1} \theta^{*}$;

- for $i=1, \ldots, n, k=1, \ldots, T$, generate $W_{k}^{(i)} \sim N(0,1)$;

- simulate $X_{k+1}^{(i)}=X_{k}^{(i)}+\left(\mu X_{k}^{(i)}-c^{*}(x)\right) \frac{1}{T}+\sigma X_{k}^{(i)} \sqrt{\frac{1}{T}} W_{k}^{(i)}+\zeta^{*} X_{k}^{(i)} Z_{k}$, where the $Z$ 's are the returns of the thin stocks obtained with the first building block;

- compute the optimal path $\mathbf{X}=\left(X_{1}, \ldots, X_{T}\right)$ of the portfolio value, where

$$
X_{k}=\frac{1}{n} \sum_{i=1}^{n} X_{k}^{(i)}, \quad \forall k=1, \ldots, T
$$

- set $\mathbf{c}=\left(\bar{c}_{1}, \ldots, \bar{c}_{T}\right)$, where

$$
\bar{c}_{h}=c^{*}\left(X_{h}\right), \quad h=1, \ldots, T .
$$

The fourth building block consists in the comparison between the solutions of the proposed model and the classical Merton's portfolio. Firstly, using the same set of parameters, the optimal Merton's portfolio value $X_{t}^{(M)}$, with $\zeta^{*}=0$, is simulated and, then, the difference process $\Delta_{t}=X_{t}^{(M)}-X_{t}$ is computed for each $\lambda$-level. The Merton's optimal controls are denoted as $\theta_{M}^{*}$ and $c_{M}^{*}$.

We perform $n=10,000$ replications of the following algorithm:

- set $\mu^{(M)}=\left(1-\theta_{M}^{*}\right) r+\mu_{1} \theta_{M}^{*}$;

- set $\sigma=\sigma_{1} \theta_{M}^{*}$;

- $X_{k+1}^{(M, i)}=X_{k}^{(M, i)}+\left(\mu X_{k}^{(M, i)}-c_{M}^{*}(x)\right) \frac{1}{T}+\sigma X_{k}^{(M, i)} \sqrt{\frac{1}{T}} W_{k}^{(i)}$, where $W_{k}^{(i)} \sim N(0,1)$ are the same set of random draws generated in the third building block;

- compute the optimal path $\mathbf{X}^{M}=\left(X_{1}^{(M)}, \ldots, X_{T}^{(M)}\right)$ of the portfolio value, where

$$
X_{k}^{(M)}=\frac{1}{n} \sum_{i=1}^{n} X_{k}^{(M, i)}, \quad \forall k=1, \ldots, T ;
$$

- compute the optimal path $\mathbf{c}^{M}=\left(\bar{c}_{1}^{(M)}, \ldots, \bar{c}_{T}^{(M)}\right)$ of the consumption, where

$$
\bar{c}_{M, h}=c_{M}^{*}\left(X_{h}^{(M)}\right), \quad h=1, \ldots, T ;
$$

- set $\Delta \mathbf{X}_{k}=X_{k}^{(M)}-X_{k}$ and $\Delta \mathbf{c}_{k}=\bar{c}_{k}^{(M)}-\bar{c}_{k}$ for each $k=1, \ldots, T$.

The optimal controls resulting from the application synthesized above are reported in Table 1 . The results show that an investor with relative risk aversion $(1-\gamma)=0.1$ and in presence of an asset characterized by low liquidity should allocate her/his wealth on the risky asset and assume a short position on the thin stock and risk-free bond. It is worth to notice that the level of the short position on thin stock decreases as the average frequency of the jumps increases. At the same time, the wealth invested in the liquid asset is constant as expected. Even though the short position on the risk free asset is quite sta-

Table 1

Optimal control triple by different levels of $\lambda$.

\begin{tabular}{lllll}
\hline & $\lambda=5$ & $\lambda=15$ & $\lambda=30$ & \multicolumn{1}{c}{$\lambda=100$} \\
\hline$\xi^{*}(x)$ & $-0.507^{* *}$ & $-0.361^{* *}$ & $-0.174^{* *}$ & $-0.083^{* *}$ \\
$\theta^{*}(x)$ & 22.222 & 22.222 & 22.222 & 22.222 \\
$\left(1-\theta^{*}(x)-\xi^{*}(x)\right)$ & -20.715 & -20.861 & -21.048 & -21.139 \\
Merton's Portfolio & & & \\
$\theta_{M}^{*}(x)$ & 22.222 & & \\
$\left(1-\theta_{M}^{*}(x)\right)$ & -21.222 & & \\
\hline
\end{tabular}

** Average estimation error $=1.69 \mathrm{E}-06$. 
ble, it can be noticed a slight decrease in the share of wealth short on the riskless bond, with respect to the frequency of the jumps in thin stock. These results are due to the assumed high degree of the investor's relative risk aversion.

The comparison with Merton's portfolio shows that the wealth allocated in the risky asset is constant and remains unchanged with respect to the proposed model. Therefore, the introduction of a light stock affects only the optimal level of allocation in the risk free asset.

In Figs. 1 and 2 the difference processes, $\Delta \mathbf{X}_{k}$ and $\Delta \mathbf{c}_{k}$, for $\lambda=5,15,30$ are depicted. They show that, in this cases, Merton's model performs slightly better than the model with thin stock even though it is worth to notice that the differences are not so relevant.

In Figs. 3 and 4 the optimal paths of wealth and consumption are reported, both for Merton's model and the proposed one in the case of $\lambda=100$. By visual inspection it is clear that the proposed model performs much more better than the Merton's one. This result overturns the ones shown in Figs. 1 and 2 for lower levels of $\lambda$. The inversion is essentially due to the reduction in the short position on the thin stock and the higher level in the frequency of jumps that let the dynamics of the thin stock be closer to those of the frequently traded risky asset.

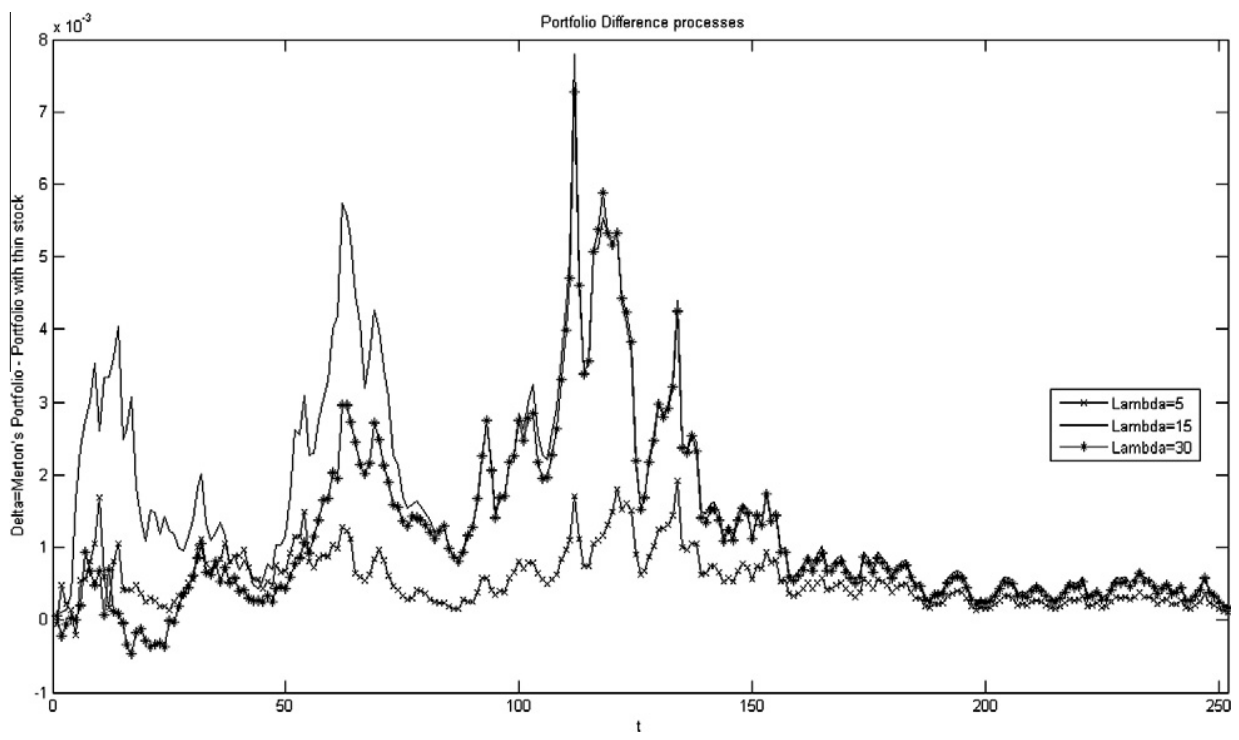

Fig. 1. Portfolio difference processes with $\lambda=5,15,30$.

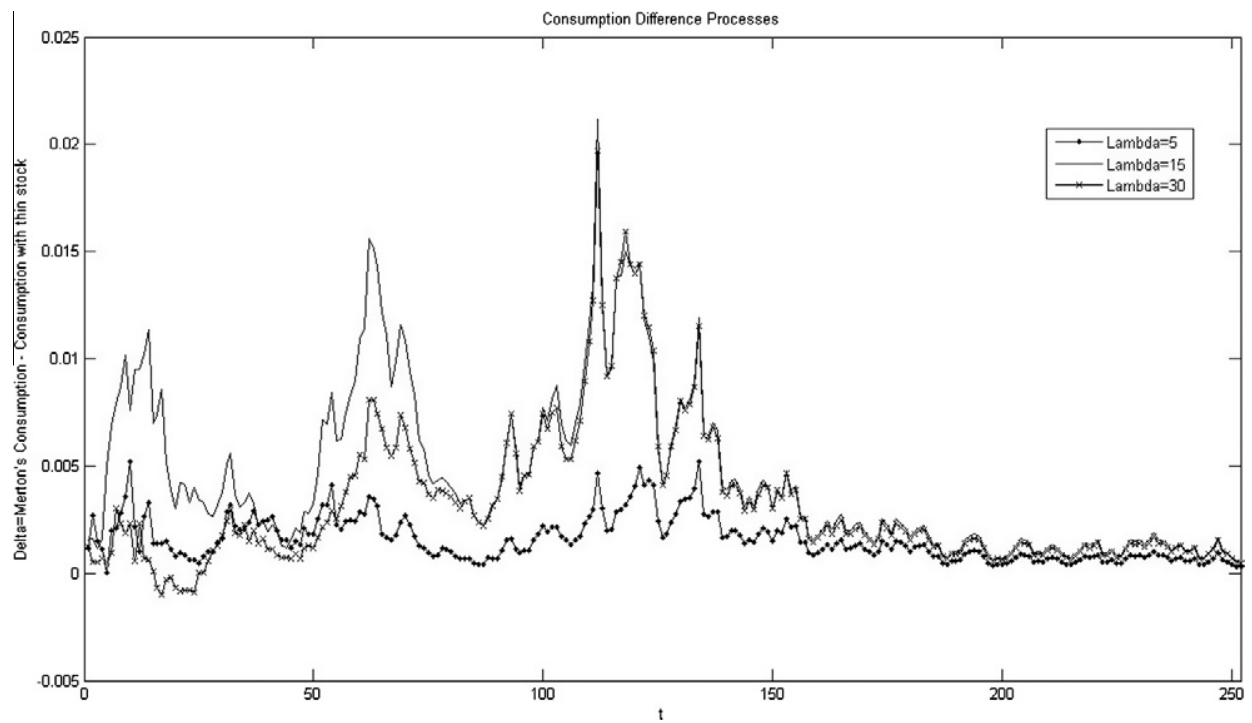

Fig. 2. Consumption difference process with $\lambda=5,15,30$. 


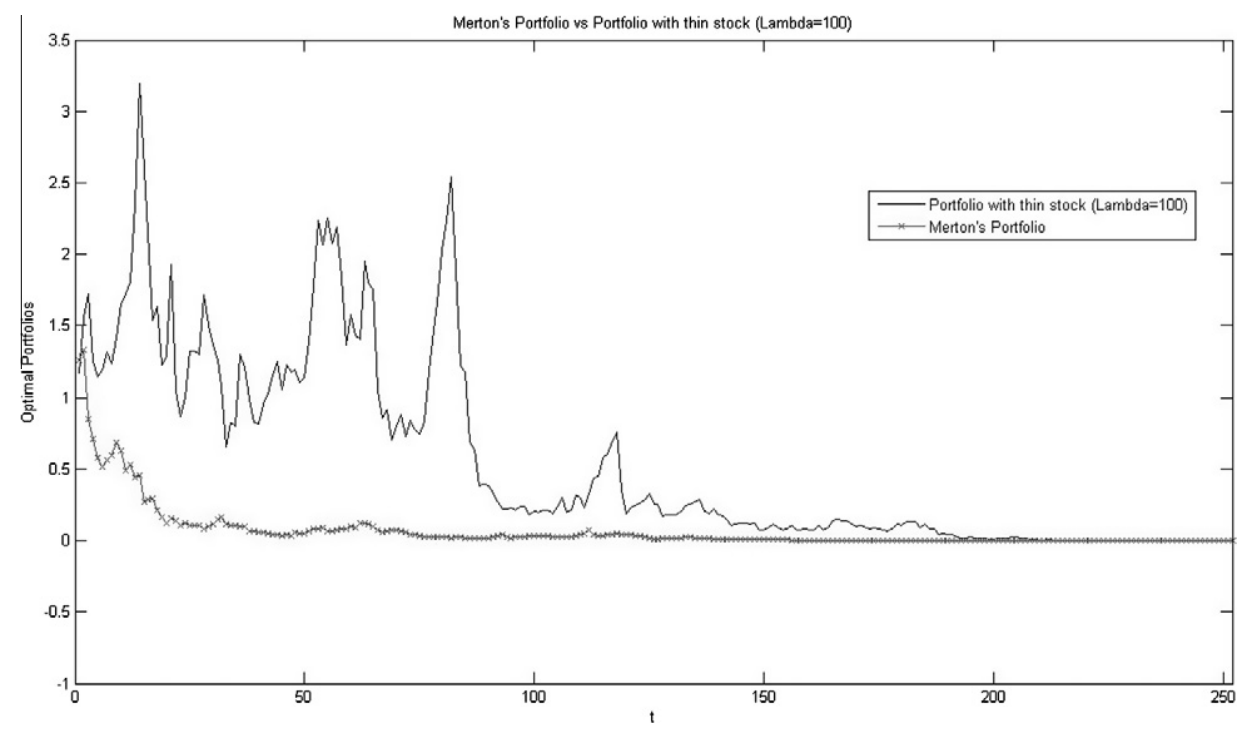

Fig. 3. Optimal wealths with $\lambda=100$.

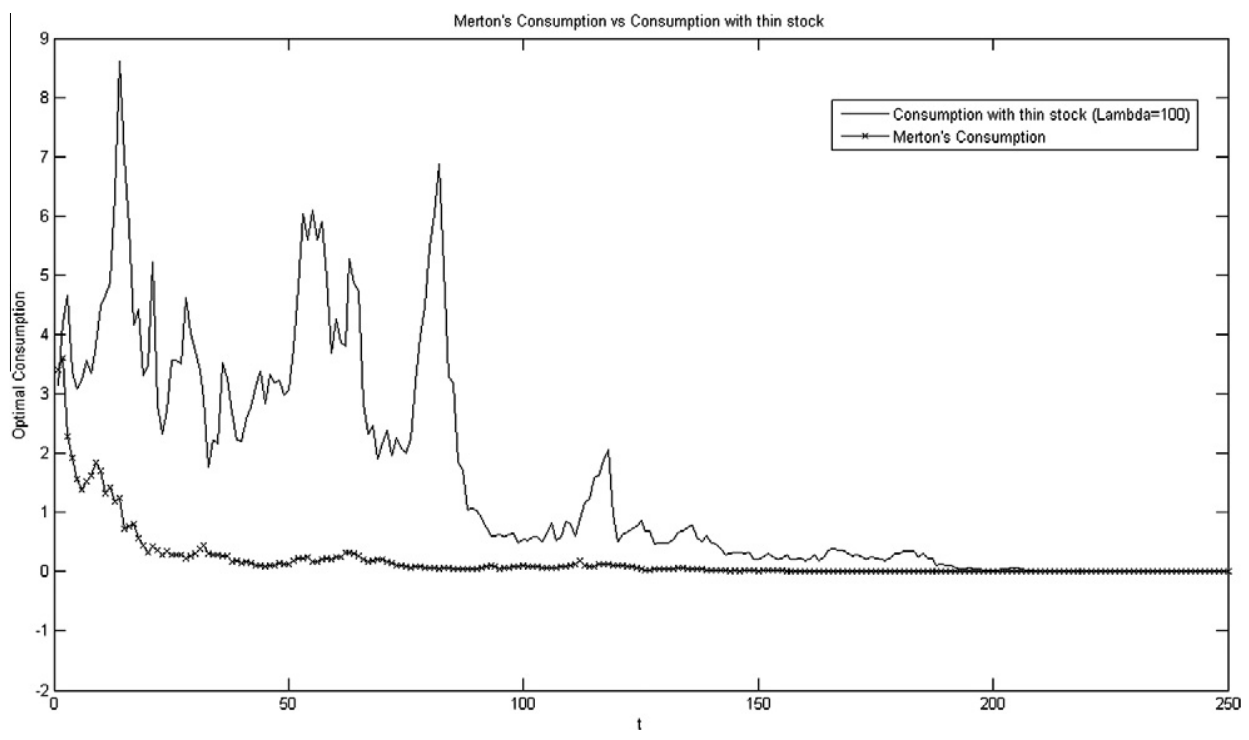

Fig. 4. Optimal consumptions with $\lambda=100$.

\section{Conclusion}

This paper deals with optimal consumption/investment choices in presence of thin stocks. Since the main characteristic of thin stocks is given by low-liquidity, a jump-type process with random times is particularly suited to describe their returns and provide a realistic model.

After developing the model in a very general framework, by assuming a power-type utility function an explicit closed form solution for the stochastic control problem is provided. In doing this, we ensure that the proposed model can be consistently compared with the classical [13]'s one.

Numerical analysis shows that the results of the proposed model are rather in line with the ones of the Merton's portfolio. Moreover the portfolio model with thin stock performs better than the Merton's one in cases of frequent jumps in light asset returns.

Probably, the most challenging extension of the proposed model is related with the analysis of the regularity of the value function in the general setting through the introduction of the HJB solution in viscosity sense. However, this could imply 
sophisticated functional analysis techniques making difficult the comparison with other standard asset allocation models. We leave this topic to future research.

\section{Appendix A. Proof of Proposition 7}

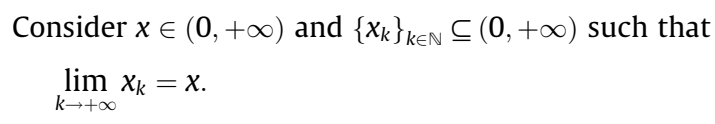

Since $(0,+\infty)$ is an open set, then $\exists r>0$ such that $B_{r}(x)=(x-r, x+r) \in(0,+\infty)$. Consider $\alpha \in(0, r)$ and $A \subset B_{r}(x)$ defined as:

$$
A=\{z \in(0,+\infty):|z-x|=\alpha\} .
$$

Since $x_{k}$ converges to $x$ as $k \rightarrow+\infty$, then $\exists \bar{K}$ large enough such that

$$
\forall k>\bar{K} \Rightarrow\left|x_{k}-x\right|<\alpha .
$$

Therefore, for each $k>\bar{K}$ and $\theta_{k} \in(0,1)$, there exists $z_{k} \in A$ such that

$$
x_{k}=\theta_{k} x+\left(1-\theta_{k}\right) z_{k} \text {. }
$$

Since $\left|z_{k}-x\right|=\alpha>0$, by (31) and (32) we obtain

$$
\lim _{k \rightarrow+\infty} \theta_{k}=1 \text {. }
$$

The concavity of $V$ implies

$$
V\left(x_{k}\right)=V\left(\theta_{k} x+\left(1-\theta_{k}\right) z_{k}\right) \geqslant \theta_{k} V(x)+\left(1-\theta_{k}\right) V\left(z_{k}\right) .
$$

Therefore, by (33):

$$
\liminf _{k \rightarrow+\infty} V\left(x_{k}\right) \geqslant 1 \cdot V(x)=V(x) .
$$

On the other hand, for each $k>\bar{K}$ and $\lambda_{k} \in(0,1)$, there exists $\omega_{k} \in A$ such that

$$
x=\lambda_{k} x_{k}+\left(1-\lambda_{k}\right) \omega_{k}
$$

Also in this case, since $\left|\omega_{k}-x\right|=\alpha>0$, by (31) and (36) we obtain

$$
\lim _{k \rightarrow+\infty} \lambda_{k}=1
$$

The concavity of $V$ implies

$$
V(x)=V\left(\lambda_{k} x_{k}+\left(1-\lambda_{k}\right) \omega_{k}\right) \geqslant \lambda_{k} V\left(x_{k}\right)+\left(1-\lambda_{k}\right) V\left(\omega_{k}\right) .
$$

Therefore, by (37):

$$
V(x) \geqslant \limsup _{k \rightarrow+\infty} V\left(x_{k}\right)
$$

By (35) and (39) we obtain

$$
\lim _{k \rightarrow+\infty} V\left(x_{k}\right)=V(x)
$$

i.e. the continuity of $V$ on $(0,+\infty)$.

\section{References}

[1] K. Aase, Optimal portfolio diversification in a general continuous-time model, Stoch. Process. Appl. 18 (1984) 81-98.

[2] O.E. Barndorff-Nielsen, Processes with normal inverse Gaussian type, Finance Stoch. 1 (1998) 41-68.

[3] R. Cont, P. Tankov, Financial Modelling with Jump Processes, Chapman \& Hall/CRC Press, Boca Raton, 2003.

[4] A. Cretarola, F. Gozzi, H. Pham, P. Tankov, Optimal consumption policies in illiquid markets, Finance Stoch. 15 (1) (2011) $85-115$.

[5] E. Eberlein, Application of generalized hyperbolic Levy Motion to finance, in: O.E. Barndorff-Nielsen, T. Mikosch, S.I. Resnick (Eds.), Levy Processes: Theory and Applications, Birkhäuser, Boston, 2001, pp. 319-336.

[6] E. Eberlein, U. Keller, Hyperbolic distributions in finance, Bernoulli 1 (1995) 281-299.

[7] D. Easley, N.M. Kiefer, M. O’Hara, J.B. Paperman, Liquidity information, and infrequently traded stocks, J. Finance 51 (1996) $1405-1436$.

[8] N.C. Framstad, B. Oksendal, A. Sulem, Optimal consumption and portfolio in a jump diffusion market, in: A. Shiryaev, A. Sulem (Eds.), Mathematical Finance, INRIA, Paris, 1998, pp. 8-20.

[9] N.C. Framstad, B. Oksendal, A. Sulem, Optimal consumption and portfolio in a jump diffusion market with proportional transaction costs, J. Math. Econ. 35 (2001) 233-257.

[10] H. Markowitz, Portfolio selection, J. Finance 7 (1) (1952) 77-91.

[11] K. Matsumoto, Optimal portfolio of low liquid assets with a log-utility function, Finance Stoch. 10 (1) (2006) $121-145$.

[12] R.C. Merton, Lifetime portfolio selection under uncertainty: the continuous-time case, Rev. Econ. Stat. 51 (1969) $247-257$.

[13] R.C. Merton, Optimal consumption and portfolio rules in a continuous time model, J. Econ. Theory 3 (1971) $373-413$.

[14] B. Oksendal, A. Sulem, Applied Stochastic Control of Jump Diffusions, second ed., Springer-Verlag, Berlin Heidelberg, 2007. 
[15] H. Pham, P. Tankov, A model of optimal consumption under liquidity risk with random trading times, Math. Finance 18 (2008) $613-627$.

[16] P. Protter, Stochastic Integration and Differential Equations, second ed., Springer-Verlag, Berlin Heidelberg, 2003.

[17] C. Rogers, The relaxed investor and parameter uncertainty, Finance Stoch. 5 (2) (2001) 131-154.

[18] C. Rogers, O. Zane, A simple model of liquidity effects, in: K. Sandmann, P. Schoenbucher (Eds.), Advances in Finance and Stochastics: Essays in Honour of Dieter Sondermann, Springer-Verlag, Berlin Heidelberg, 2002, pp. 161-176.

[19] P.A. Samuelson, Lifetime portfolio selection by dynamic stochastic programming, Rev. Econ. Stat. 51 (1969) 239-246.

[20] W. Schoutens, Levy Processes in Finance, Wiley, 2003.

[21] Y.H. Shin, B.H. Lim, U.J. Choi, Optimal consumption and portfolio selection problem with downside consumption constraints, Appl. Math. Comput. 188 (2007) 1801-1811. 